\title{
UNREASONABLE IMPLICATIONS OF REASONABLE IDIOTYPIC NETWORK ASSUMPTIONS
}

\author{
- Rob J. De Boer and Pauline Hogeweg \\ Bioinformatics Group, \\ University of Utrecht, \\ Padualaan 8, 3584 CH Utrecht, \\ The Netherlands
}

We first analyse a simple symmetric model of the idiotypic network. In the model idiotypic interactions regulate B cell proliferation. Three non-idiotypic processes are incorporated: (1) influx of newborn cells; (2) turnover of cells; (3) antigen. Antigen also regulates proliferation.

A model of 2 B cell populations has 3 stable equilibria: one virgin, two immune. The twodimensional system thus remembers antigens, i.e. accounts for immunity. By contrast, if an idiotypic clone proliferates (in response to antigen), its anti-idiotypic partner is unable to control this. Symmetric idiotypic networks thus fail to account for proliferation regulation.

In high-D networks we run into two problems. Firstly, if the network accounts for memory, idiotypic activation always propagates very deeply into the network. This is very unrealistic, but is an implication of the "realistic" assumption that it should be easier to activate all cells of a small virgin clone than to maintain the activation of all cells of a large (immune) clone. Secondly, graph theory teaches us that if the (random) network connectance exceeds a threshold level of one interaction per clone, most clones are interconnected. We show that this theory is also applicable to immune networks based on complementary matching idiotypes. The combination of the first "percolation" result with the "interconnectance" result means that the first stimulation of the network with antigen should eventually affect most of the clones. We think this is unreasonable.

Another threshold property of the network connectivity is the existence of a virgin state. A gradual increase in network connectance eliminates the virgin state and thus causes an abrupt change in network behaviour. In contrast to weakly connected systems, highly connected networks display autonomous activity and are unresponsive to external antigens. Similar differences between neonatal and adult networks have been described by experimentalists.

The robustness of these results is tested with a network in which idiotypic inactivation of a clone occurs more generally than activation. Such "long-range inhibition" is known to promote pattern formation. However, in our model it fails to reduce the percolation, and additionally, generates semi-chaotic behaviour. In our network, the inhibition of a clone that is inhibiting can alter this clone into a clone that is activating. Hence "long-range inhibition" implies "long-range activation", and idiotypic activation fails to remain localized.

We next complicate this model by incorporating antibody production. Although this "antibody" model statically accounts for the same set of equilibrium points, it dynamically fails to account for state switching (i.e. memory). The switching behaviour is disturbed by the autonomous slow decay of the (long-lived) antibodies. After antigenic triggering the system now performs complex cyclic behaviour. Finally, it is suggested that (idiotypic) formation of antibody complexes can play only a secondary role in the network.

In conclusion, our results cast doubt on the functional role of a profound idiotypic network. The network fails to account for proliferation regulation, and if it accounts for memory phenomena, it "explodes" upon the first encounter with antigen due to extensive percolation. 
1. Introduction. Previously we (De Boer, 1988; De Boer and Hogeweg, 1988) analysed a simple, symmetric, model of the idiotypic immune network. This analysis demonstrated that suppressive (i.e. negative) interactions could not play a role in such networks. This suggested that the model could be further simplified by omitting negative interactions and that this would not alter the results. Having verified this hypothesis, we here proceed with the analysis of our symmetric model by investigating networks consisting of many, instead of a few (De Boer and Hogeweg, 1988), different clones of lymphocytes.

We view idiotypic network theory (Jerne, 1974) as a straightforward extension of the normal scheme of immune systems. Immune systems consist of an extremely large $\left(>10^{7}\right)$ number of different lymphocyte clones. The lymphocytes of each clone all share an identical (unique) antigen receptor. The generation of the variable regions of these receptors involves random processes such as somatic recombination and mutation (Early et al., 1980; Berek et al., 1985). The primary repertoire of antigen receptors can therefore be visualized as a large (possibly) random array of receptor molecules. Antigens, i.e. external structures, match complementary receptor molecules. Because of the overwhelming diversity of receptors, each (possibly random) antigen is expected to match at least one of the $\left(10^{7}\right)$ receptor molecules. The clones that recognize (i.e. match to) the antigen proliferate and develop effector functions. Idiotypic network theory (Jerne, 1974) states that if the random repertoire of receptors can recognize any random antigen, receptors should also be able to recognize other receptors. The structure (i.e. antigen) presented by a receptor is called the "idiotype" (Jerne, 1974); the interaction between receptors is referred to as an "idiotypic" interaction. Via idiotypic interactions clones should thus be able to interact in a stimulatory and/or inhibitory manner. The collection of idiotypic interactions defines a profound idiotypic network of a complexity comparable to that of the neural network (Jerne, 1974; Hoffmann, 1986).

Neural networks, for which the Hopfield networks are the general paradigm system, are capable of complex computational processes (such as learning and memory) (Hopfield and Tank, 1986). Neural networks have these properties if connection strengths evolve following certain "learning rules", such as the one proposed by Hebb (1949). Interestingly, idiotypic connections might evolve in a similar way in immune networks if idiotypic interactions become more specific by affinity maturation (Weisbuch, 1989). Moreover, the "artificial" symmetry assumption of neural networks (Hopfield and Tank, 1986) is very natural in idiotypic networks (Hoffmann, 1979, 1980; De Boer and Hogeweg, 1988). Thus, via its network properties, the immune system might perform very complex tasks. Additionally, because lymphocytes are reproducing entities that use random mutation (but not crossing over) as a "genetic operator", immune systems also bear resemblance to classifier systems (Holland, 1986; Farmer et al., 1986). Classifier systems are very powerful learning systems. 
Whether or not immune systems function [i.e. compute (Segel and Perelson, 1988)] by such cognitive (Varela et al., 1988) network properties however remains an open question. In the first place, we think that even if it were to be demonstrated that idiotypic network models have learning or cognitive properties this does not necessarily prove that specific immunological phenomena (e.g. immunity) are indeed due to "cognition". Even if immune systems are cognitive, this might still not mean that they are able to solve the problem of, for instance, antigen specific immunity. Several authors have argued that idiotypic interactions cannot play a functional role (Cohn, 1986; Langman and Cohn, 1986). Furthermore, we have shown (De Boer and Hogeweg, 1986, 1987a, 1987b; De Boer, 1989) that immune systems perform complex tasks by means of basic interactions at the level of clonal selection, i.e. by non-idiotypic interactions. However, because idiotypic interactions seem to be inevitable (because of the extensive repertoire of receptors), we here investigate the behaviour that can be generated by networks of such basic idiotypic interactions. Immunity and/or cognition is therefore a possible outcome of our analysis.

In order to avoid incorporating ambiguous assumptions, we here investigate a very simple model of the idiotypic network. Its simplicity enables us to analyse an idiotypic network theory that is based on only the most fundamental immunological assumptions. We thus investigate whether "fundamental" idiotypic interactions can indeed account for the variety of immunological phenomena for which networks are claimed to be responsible (see for instance: Jerne, 1974; Bona and Pernis, 1984). We here concentrate on two such phenomena: (1) immunological memory (immunity); (2) the control of (excessive) proliferation. Intuitively, the idiotypic control of proliferation is visualized as an inhibitory interaction between the proliferating clone and its anti-idiotypic partner(s). The existence of multiple stable equilibria in a network system seems to provide an (intuitive) explanation for memory phenomena: in response to different antigens networks switch to different equilibria.

2. The Assumptions. Symmetric network theory has demonstrated (Hoffmann, 1979, 1980; De Boer, 1988; De Boer and Hogeweg, 1988) that lymphocyte populations exert "helper" or "suppressor" functions depending on the idiotypic circumstances. As a consequence, it seems sufficient to consider only one class of cells, here clones of B-lymphocytes. Moreover, interactions are symmetric and are therefore not exclusively stimulatory or inhibitory. Hence, the two parameters that determine the magnitude (and possibly the sign) of idiotypic interactions are: (1) the affinity; (2) the respective population densities (De Boer, 1988).

We assume that all B-lymphocyte populations are regulated by three 
processes: (1) influx of newborn cells from the bone marrow; (2) normal turnover (decay) of cells; (3) proliferation. It is further assumed that idiotypic interactions influence the rate of cell proliferation. We consider only B cells in this paper, i.e. we implicitly assume that these idiotypic B cell interactions are $T$ cell independent or that $\mathrm{T}$ cell help is always sufficient. Additionally, we lump the clones of B cells and the antibody molecules that they produce into one population. Because the lifespan of B cells and antibodies is different we relax this simplifying assumption in the last section. Elsewhere (De Boer and Hogeweg, 1989) we investigate the effects of T cells.

The network consists of $\mathrm{N}$ clones, each of which is only identified by a unique random receptor. A clone $\left(X_{i}\right)$ recognizes another clone $\left(X_{j}\right)$ if (part of) their respective receptors can be matched complementarily (Fig. 1). The accuracy of this match specifies the affinity $\left(\mathbf{A}_{i j}\right)$ of the idiotypic interaction between $X_{i}$ and $X_{j}\left(0 \leqslant \mathbf{A}_{i j} \leqslant 1\right)$. Each clone can also be stimulated by antigen $\left(A g_{i}\right)$. Antigen cannot grow and is either removed by the clones or by us.

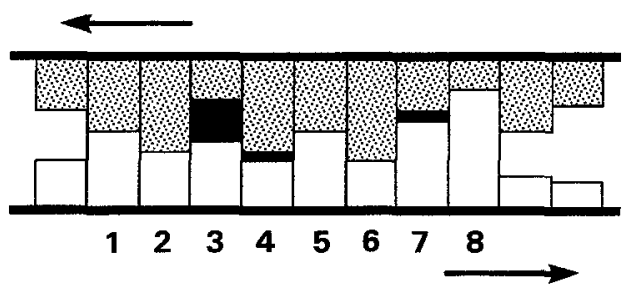

Figure 1. Complementary matching. Two idiotypes, i.e. the white and the shaded pattern, are shifted into each other until they collide (here at position 1,2,5,6, and 8). The remaining distance (i.e. the black area) is a measure for the affinity. The patterns are shifted along each other (indicated by the arrows) in order to find the place were the match is most perfect. The match is measured over a distance of eight adjacent positions.

2.1. Influx/efflux. In the absence of idiotypic interactions the clone size is determined solely by the balance between the source $\left(S_{i}\right)$ of cells from bone marrow and the death $(D)$ of cells in the periphery. This suffices for a stable virgin state at a clone size of $S / D$. Because the influx of cells per clone is small, and the rate of cell turnover is high, virgin populations are typically small. In the virgin state idiotypic interactions are therefore low or absent. This contrasts strongly with the assumptions of previous verbal (Jerne, 1974) and mathematical (Hoffmann, 1979; Gunther and Hoffmann, 1982) models; in these models it is assumed that idiotypic interactions are suppressive in the virgin state. We demonstrated previoussly however that our models are more powerful (De Boer, 1988; De Boer and Hogeweg, 1988). Moreover simple 
influx and decay processes play a pivotal role in "clonal selection" models of proliferative immune reactions (De Boer and Hogeweg, 1986, 1987a,b).

2.2. Symmetry. If idiotypic recognition is based on complementary matching and receptor crosslinking, idiotypic interactions are necessarily symmetric. If idiotype " $i$ " matches " $j$ ", " $j$ " should also match " $i$ "; if " $i$ " antibodies crosslink " $j$ " receptors, " $j$ " antibodies can do the same. Hoffmann $(1979,1980)$ first proposed this simple and attractive symmetry theory; Hoffmann (1980) and Jerne (1984) review empirical data that support symmetry theory (Jerne's "preferred partners"). Note that, because recognizing and being recognized are assumed to be identical, symmetry disposes of the distinction between "paratope" and "idiotype" (Hoffmann, 1980). Note also that complementary matching of parts of the idiotype implies that idiotypes can be matched onto themselves. We omit such interactions (i.e. we set all $\mathbf{A}_{i i}$ to zero). In Section 4.3 we will assume that inhibitory interactions occur more generally, i.e. require a lower affinity, than stimulatory interactions. The additional inhibition matrix (B) is also symmetric.

2.3. Dose-response relation. Idiotypic interactions can be stimulatory (Eichmann and Rajewsky, 1975; Vakil and Kearny, 1986; Bernabe et al., 1981) and inhibitory (Hardt et al., 1972; Eichmann, 1974; Vakil and Kearny, 1986). Because B cells are most probably activated by the crosslinking of the antigen receptors (surface Ig) (Abbas, 1988), it is to be expected that the rate of cell activation increases if the concentration of the crosslinking agent (here antigen or anti-idiotypic antibody) increases. However, when these concentrations become too high the efficacy of cell activation by receptor crosslinking decreases (Perelson, 1984). Thus, the present model is based on the efficacy of cell activation and proliferation induction (by receptor crosslinking); inhibitory interactions are incorporated as the reduction of the proliferation rate. This argument corresponds to a (log) bell-shaped proliferation dose response curve (see e.g. Fig. 2D), i.e. to the kind that can be found in any immunology textbook. Despite this simplicity, previous models incorporated a totally different (Hoffmann, 1980) or even reverse dose response relation [i.e. "escape from suppression" (Jerne, 1974)].

3. The Model. We consider $N$ clones of B-lymphocytes $\left(X_{i}\right)$ with an influx of $S_{i}$ cells per day from the bone marrow and a rate $D$ of cell turnover. Clones proliferate in response to antigen $\left(A g_{i}\right)$ and to the total of anti-idiotypic clones $\left(\alpha l d_{i}\right)$. The strength of the idiotypic interaction is determined by the affinity matrix $\mathbf{A}$. This matrix is symmetric (all $\mathbf{A}_{i j}=\mathbf{A}_{j i}$ ); furthermore clones never recognize themselves (all $\mathbf{A}_{i i}=0$ ). The rate of cell proliferation is governed by a growth dose response function $G\left(X_{i}, A g_{i}\right.$, ald $)$, which depends on: (i) the size of 

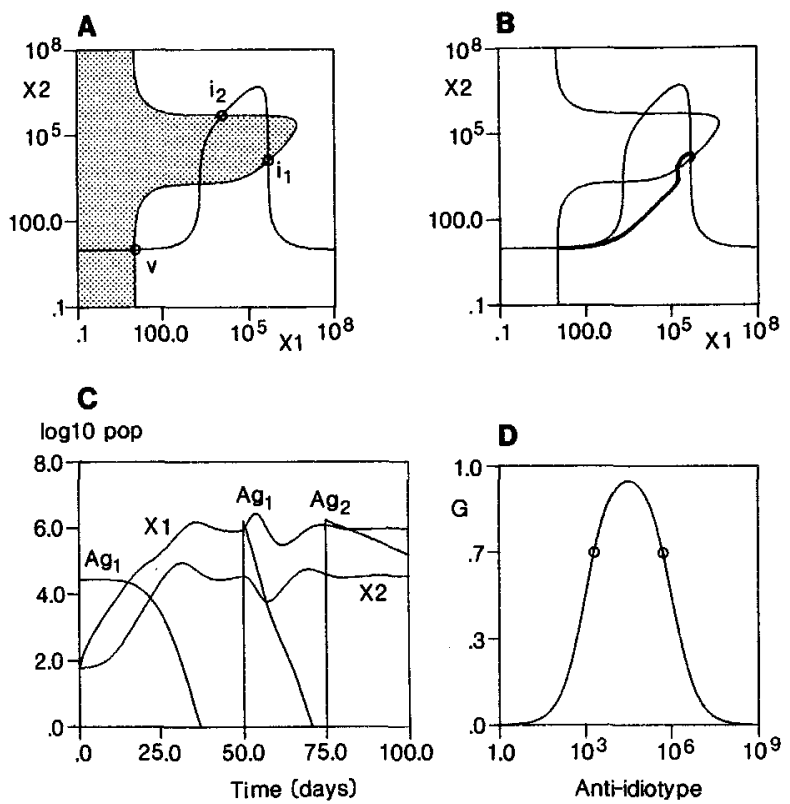

Figure 2. A two-dimensional network with maximum affinity $\left(\mathbf{A}_{12}=1\right)$. (A) The $X_{1}^{\prime}=0$ and $X_{2}^{\prime}=0$ isoclines define 3 stable equilibria: a virgin state (V) and two immune states $\left(I_{1}\right.$ and $\left.I_{2}\right)$. The $X_{1}^{\prime}>0$ region is shaded. (B) The trajectory of a switch from the virgin state to an immune state $\left(I_{1}\right)$ as it is evoked by an antigen dose of $A g_{1}=10^{4}$ cells. (C) At day zero, in the virgin state, $A g_{1}$ is introduced in a dose of $10^{4}$ cells (see Fig. 2B); it is reintroduced in a dose of $10^{6}$ cells at day $50 ; \mathrm{Ag}_{2}$ is introduced in such a dose at day 75. In the $I_{1}$ state $X_{1}$ is immune; $X_{2}$ is suppressed. (D) the (log) bell-shaped dose-response curve $(\mathrm{G})$ as a function of the anti-idiotype. The circles indicate the location of the immune and suppressed clone respectively.

the clone (a buffering term); (ii) the antigen; (iii) the total amount of antiidiotype $\left(\alpha l d_{i}\right)$. The function $G\left(X_{i}, A g_{i}, \alpha l d_{i}\right)$ is maximally one; proliferation per cell then proceeds at a rate $P$ per day. The anti-idiotype dose response function $(G)$ is a $(\log )$ bell-shaped curve (Fig. 2D), i.e. anti-idiotypic antibody concentrations that are too large inhibit the crosslinking and hence the rate of cell proliferation. Antigen can only increase (up to a certain maximum) the rate of cell proliferation: the antigen dose response curve is a simple saturation function. (The results remain similar if proliferation induced by antigen also fcllows this bell-shaped dose response curve.)

$$
\begin{gathered}
\alpha l d_{i}=\sum_{j=1}^{N} \mathbf{A}_{i j} X_{j} \\
G\left(X_{i}, A g_{i}, \alpha l d_{i}\right)=\frac{A g_{i}+\alpha l d_{i}}{P_{1}+F X_{i}+A g_{i}+\alpha l d_{i}} \times \frac{P_{2}}{P_{2}+\alpha l d_{i}}
\end{gathered}
$$




$$
\frac{\mathrm{d} X_{i}}{\mathrm{~d} t}=S_{i}-D X_{i}+P X_{i} G\left(X_{i}, A g_{i}, \alpha l d_{i}\right)
$$

Antigen cannot grow and is either removed by the clone that recognizes it $\left(X_{i}\right)$, or is incorporated as a constant $(K=0)$ :

$$
\frac{\mathrm{d} A g_{i}}{\mathrm{~d} t}=-\frac{K A g_{i} X_{i}}{K_{1}+X_{i}} .
$$

3.1. Parameters. The parameter setting is: $S \approx 10$ cells day $^{-1} ; D=1$ day $^{-1}$; $P=1.5$ day $^{-1} ; P_{1}=10^{3} ; P_{2}=10^{6} ; F=0.01, K=1, K_{1}=10^{5}$. Buffering ensures that large $X_{i}$ populations $\left(F X_{i} \approx P_{1}\right)$ cannot be stimulated by small antigen and/or anti-idiotype concentrations (De Boer and Hogeweg, 1988). The virgin population density equals $S / D \approx 10$ cells. The influx is slightly different for each clone (to prevent settlement into unstable equilibria): $S$ has a mean of 10 cells per day with a $10 \%$ standard deviation. Virgin populations are too small to evoke proliferation $\left(S / D \ll P_{1}\right)$ : idiotypic interactions are negligible in the virgin state. Maximum proliferation proceeds at a rate $P-D=0.5$ cells per cell per day (this corresponds to a doubling time of about $16 \mathrm{hr}$ ).

3.2. Previous models. The parameters of the present model are slightly different from those used before (De Boer, 1988; De Boer and Hogeweg, 1988). In the previous model antigen grows exponentially; because we required that such an antigen could be rejected by the network we imposed some restrictions on the parameter values. We now opt for somewhat simpler functions and parameter values. We: (1) omit negative (suppressive) interactions; (2) no longer use sigmoid dose response curves; (3) reduce the life-time of cells to one day; (4) reduce the influx of virgin cells (2-fold). Negative interactions were omitted because in the previous model they failed to play a role. The incorporation of sigmoid dose response curves generated a more pronounced difference between proliferation and suppression and hence facilitated antigen rejection. Finally, the present model has a lower virgin state; which is therefore more easily preserved (De Boer, 1988).

3.3. Methods. The affinity matrix is either based on a complementary matching algorithm, or is drawn randomily from a uniform distribution between zero and one. The complementary matching algorithm resembles that of Farmer et al. (1986) and Perelson (1988). Ours matches patterns (landscapes) defined by an array of real numbers (that are uniformly distributed between zero and one), see Fig. 1. We measure the total distance within each adjacent set of (here 8) numbers (i.e. the "mask", e.g. an idiotope). Patterns are shifted along each other in order to find the best possible match. If 
this minimum total distance is larger than one, the affinity between the two patterns is zero. Otherwise it is one minus this distance (i.e. $0 \leqslant \mathbf{A}_{i j} \leqslant 1$ ). This procedure generates an exponential distribution of affinities (i.e. high affinity interactions are rare); this seems realistic. In order to vary the network connectance, we vary the length of the pattern (i.e. the number of idiotopes per idotype). Because we only want to vary the distribution of connections, and not their magnitude, affinity matrices are re-scaled afterwards. The maximum affinity is set to one, the rest is scaled proportionally.

Following the usual convention, we talk of antibodies in levels. The $A b_{1}$ level $\left(X_{1}\right)$ recognizes the antigen, $A b_{2}$ clones $\left(X_{2}\right)$ recognize (and are recognized by) $A b_{1}$ clones, and so on. The actual level of a clone is determined by the lowest antibody level with which it interacts (whatever the affinity); this argument represents the affinity matrix as a tree (i.e. omits cycles of interactions). The low-D models have been analysed by GRIND (De Boer, 1983) which performs numerical 0 -isocline analysis, and numerical integration by means of ROW4A (Gottwald and Wanner, 1982). High-D models are integrated by means of a variable step size Runge-Kutta-Merson integrator (D02BBF) implemented in NAG (1984).

\section{Results}

4.1. Memory but no suppression in two-dimensional networks. In Fig. 2 we analyse a two-dimensional network of two clones that see each other with maximum affinity $\left(\mathbf{A}_{12}=1\right)$. (Similar results were described before (De Boer, 1988) for a somewhat different model.) The qualitatively different regions of idiotype anti-idiotype state space are indicated by the $X_{1}^{\prime}=0$ and $X_{2}^{\prime}=0$ isoclines (Fig. 2A); the $X_{1}^{\prime}>0$ region (i.e. the $X_{1}$ proliferation region) is shaded. This two-dimensional network has three stable equilibria: one virgin state and two immune states. The system is thus able to remember antigens by switching from the virgin state to one of the immune states. This is demonstrated in Figs 2B and C: clone $X_{1}$ is stimulated by antigen (in a dose of $10^{4}$ cells). $X_{1}$ proliferation commences which activates the anti-idiotypic $X_{2}$ (see also Fig. 2C, day 0-50). Both clones proliferate and settle into the $X_{1}$ immune state. In this equilibrium both clones are enlarged $\left(X_{1} \gg X_{2}\right)$. The large $X_{1}$ population accounts for rapid antigen rejection, i.e. for immunity. The same antigen $\left(A g_{1}\right)$ is reintroduced (in a large dose of $10^{6}$ cells) at day 50 (Fig. 2C): $X_{1}$ further proliferates which further suppresses $X_{2}$. The fact that $X_{2}$ really is suppressed is shown by the introduction of the same dose of $\mathrm{Ag}_{2}\left(10^{6}\right.$ cells $)$ at day 75. $X_{2}$ is relatively small and fails to react (fails to proliferate); antigen is removed very slowly.

The immune states in this model are maintained by reciprocal stimulation. Because small (virgin) clones fail to induce proliferation of the anti-idiotype, both clones have to be enlarged (i.e. have to proliferate) in the immune state. 
(This contrasts strongly with immunity maintained by (long-lived) memory cells, which is a resting state.) The essential difference between the proliferating immune clone and the proliferating suppressed clone is shown in Fig. 2D. The left circle corresponds to the immune clone, the right circle to the suppressed one. Per cell both clones proliferate at an equal rate; this is a necessity because in equilibrium proliferation should outweigh the rate of cell decay (which is identical for all cells). The only difference in the proliferation of the immune and the suppressed clone is therefore their location on the proliferation dose response curve. Further idiotypic stimulation of the immune clone leads to further proliferation, whereas further idiotypic stimulation of the suppressed clone can only derease the rate of cell proliferation.

The present model has virgin and immune states (and is thus able to remember antigens) if $\mathbf{A}_{12}>0.02$. Therefore although we have omitted an affinity threshold, i.e. a minimum affinity, the model incorporates one. This is due to the buffering parameter (De Boer and Hogeweg, 1988). If $0.02<\mathbf{A}_{12}<$ 0.08 , the model has one immune state (for both clones) and one virgin state.

Absence of proliferation regulation. Suppression (i.e. the down regulation of a large proliferating clone) is impossible in these symmetric models (De Boer, 1988). In symmetric models (where there are no "suppressor" clones) the clone stimulated by antigen always wins from the anti-idiotypic clone(s) just because it will always be larger due to its initial proliferative advantage provided by antigen. Thus proliferating clones suppress their anti-idiotypic "suppressors" long before these can become suppressive. Similar results are obtained in highD networks (not shown): the total of anti idiotypic clones is usually smaller than the effect of the proliferating idiotypic clone. We think that the absence of proliferation control seriously questions the general assumption that idiotypic interactions play an important regulatory role. Note that in our models (see De Boer and Hogeweg, 1988), idiotypic suppression easily occurs following "experimental manipulation", e.g. the artificial introduction of a large antiidiotypic clone; this gives the anti-idiotype an advantage.

For these low-D networks, we conclude that idiotypic interactions (1) can easily account for memory (immunity) phenomena by forcing switches from virgin to immune states, but (2) fail to account for proliferation regulation (suppression). Memory generated by network switches is a minimal explanation since it does not require specialized memory cells. However we think that such memory cells provide an easy alternative explanation for immunity phenomena. Moreover, life-time transitions between long-lived memory cells and short-lived effector cells play an important role in the regulation of clonal immune reactions (De Boer and Hogeweg, 1987a,b). Another minimal explanation for immunological memory would be the persistence of antigen (Gray, 1988; De Boer and Hogeweg, 1988). Moreover, the fact that symmetric 
idiotypic interactions fail to account for suppression during "non-manipulated" immune reactions supports the idea that idiotypic interactions do not play a functional role (Cohn, 1986).

\subsection{Absence of fading plus a connectivity threshold generate percolation}

Absence of fading. The two clones in the previous section could only see one idiotypic partner [this is compatible with Hoffmann's (1980) plus-minus and Jerne's (1984) preferred partner arguments]. The connectivity of idiotypic networks is probably much greater (Holmberg et al., 1984, 1986; Holmberg, 1987). On the basis of accessibility computations of a three-dimensional structure analysis of immunoglobulin molecules, Novotny et al. (1987) estimated that each molecule may be recognized by as many as 40 antiimmunoglobulin molecules. We therefore now go on to consider networks with a higher connectivity.

First consider a network in which each clone has exactly two idiotypic partners. This defines circular networks (see Fig. 3A). In order to study the propagation of signals into the network, we analysed a number of such $(100-D)$ systems. The network is first allowed to equilibrate in a virgin state (i.e. without antigen); we then introduce an antigen ( $A g_{1}$ in a constant concentration of $10^{4}$ cells) that is recognized by only one of the clones $\left(X_{1}\right)$. This antigen is removed after an (arbitrary) period of 25 days. In response to antigen $X_{1}$ proliferates, thereby activating $X_{2}$ and $X_{n}$, which also start to proliferate. Because this usually activates $X_{3}$ and $X_{n-1}$, which in turn activates $X_{4}$ and $X_{n-2}$, the signal provided by the antigenic stimulation propagates deeply into the network. On average the signal fades at level 10 (based on 10 networks each with two propagation branches). In our models, it generally takes the signal about half a year to reach level 10 . The final equilibrium state usually corresponds to an alternating sequence of immune and suppressed clones (i.e. odd-numbered clones are immune (for $N$ is even) and even-numbered clones suppressed). This is indicated in Fig. 3A by the shading of the (generally) immune clones. If cycles are short, both branches of the signal meet somewhere in the middle. This stops the propagation and results in similar equilibria. We conclude that the signal penetrates very deeply into the network; because this takes a long time it seems rather absurd. Moreover the intensity of the signal fails to reduce: clones keep on switching to the same type of immune and suppressed states.

The absence of fading is further analysed in simple 4-D networks. In these networks $X_{1}$ and $X_{2}, X_{2}$ and $X_{3}$, and $X_{3}$ and $X_{4}$ recognize each other; $X_{1}$ is stimulated by antigen; and $X_{3}$ and $X_{4}$ see each other with maximum affinity $\left(\mathbf{A}_{34}=1\right)$. If $\mathbf{A}_{23}$ is sufficiently large, and $X_{1}$ and $X_{2}$ switch to immune/suppressed, $X_{2}$ might stimulate $X_{3}$ (i.e. the anti-anti-idiotype) to switch to 

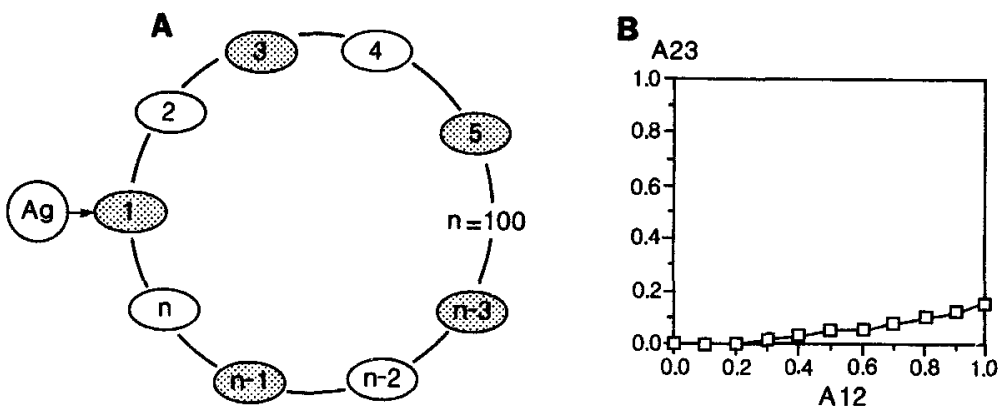

Figure 3. Networks with two connections per clone. (A) 100-D cyclic network in which clone one $\left(X_{1}\right)$ is stimulated by antigen. The shaded clones generally switch to immune. (B) The minimum affinity $\left(\mathbf{A}_{23}\right)$ between two independent twodimensional networks required for idiotypic signal to percolate. The curve is shown as function of the affinity $\left(\mathbf{A}_{12}\right)$ between the clone stimulated by antigen and its partner; the other affinity $\left(\mathbf{A}_{34}\right)$ equals one.

immune. This would force $X_{4}$ to switch to suppressed (because $\mathbf{A}_{34}=1$ ). We thus study the connectivity (i.e. $\mathbf{A}_{23}$ ) between two networks (i.e. $X_{1,2}$ and $X_{3,4}$ ) which is required for the idiotypic signal to propagate (and reach $X_{4}$ ). Figure 3B depicts the minimum $\mathbf{A}_{23}$ that is required for $X_{4}$ to switch in response to the antigenic stimulation of $X_{1}$ (as a function of $\mathbf{A}_{12}$ ). The figure shows that the affinity $\left(\mathbf{A}_{23}\right)$ that is required for $X_{4}$ to switch is much lower than the affinity $\left(\mathbf{A}_{12}\right)$ required for $X_{2}$ to switch. Thus if an antigen triggers the switching of anti-idiotypic clones, we also expect the next idiotypic level to switch (and so on for the next levels).

The verbal explanation for this result is simple. If an anti-idiotypic clone $\left(X_{2}\right.$ is able to sustain the proliferation (i.e. the immune state) of all cells of the previous, necessarily large, immune clone $\left(X_{1}\right)$, it is also able to activate the (few) cells of the clones of the next level $\left(X_{3}\right)$ because these clones are small (i.e. virgin). In the present model, the activation of all cells of a small (virgin) clone is easier, i.e. requires a smaller clone and/or affinity, than the activation of all cells of a large (immune) clone. This (realistic) assumption is thus responsible for the absence of fading.

This "absence of fading" argument no longer holds if the idiotypic network is not required to account for memory phenomena. If idiotypic interactions are too weak to push clones stimulated by antigen into an immune state, they may also be too weak to activate virgin third-level clones. However, for functional networks of B cell interactions, we conclude that idiotypic signals are expected to propagate extensively because signals fail to fade.

Connectivity thresholds. A randomly connected, symmetric, idiotypic network corresponds to a (isotrophic) random undirected graph in which 
idiotypic connections form edges and clones correspond to nodes. Connectivity properties of isotrophic random undirected graphs (in which $E$ edges connect $N$ nodes equiprobably) were analysed by Erdos and Renyi (1959, 1960), in Kauffman (1986). The following findings for random infinite graphs (Kauffman, 1986) are of interest for our (finite) random idiotype networks. If $E \ll N$ only small isolated structures are found, in which any node is connected to a few others. As the ratio of $E / N$ exceeds $1 / 2$, a threshold is reached and most nodes are interconnected in one enormous structure. As $E / N$ further increases, more isolated nodes are brought into this very large connected structure. For finite graphs these thresholds soften to sigmoids. In our idiotypic networks, an average of one edge per two clones $(E / N=1 / 2)$ corresponds to a connectance of one idiotypic partner per clone $(n c=1)$. The Erdos and Renyi theory thus predicts that one the idiotypic connectance exceeds one partner per clone, most clones suddenly become interconnected. Thus the properties of the network may suddenly change. [Such a sudden change has been described before (De Boer, 1988) but did not refer to this theory.]

High-D idiotypic networks. The combination of the Erdos and Reyni theory and our "absence of fading" findings predicts that the stimulation of one clone in a sufficiently connected network will finally affect all clones of the immune network, thus disrupting the virgin state of (nearly) all the clones. This prediction is analysed here. By analysing randomly conneted (100-D) networks (Figs 4A and C) we first confirm the applicability of the Erdos and Reyni theory to random idiotypic networks. Because biotic immune networks are not necessarily random, we additionally generate affinity matrices by the (nonrandom) complementary matching of random (idiotypic) patterns (see Fig. 1). It will be shown (Figs 4B and D) that the "threshold" connectivity properties of these "pattern" networks cannot be distinguished from those of random networks.

In both types of networks ("random" vs "pattern") we analyse the effect of matrix connectance on the system behaviour. In both networks connectance is measured by the actual number $(n c)$ of connections per clone (i.e. the number of non-zero A elements per row). In "pattern" networks the connectance is increased by elongation of the random pattern (i.e. by allowing for more idiotopes per idiotype). Because our complementary matching rule generates an exponential distribution of affinities (see Section 3 ), the average affinity of "pattern" networks is lower than that of an equally connected (uniformly distributed) "random" network. To compensate for this lower affinity between level one and level two $\left(X_{1}\right.$ and $\left.X_{2}\right)$, "pattern" networks are triggered by a higher antigen concentration $\left(10^{5}\right.$ instead of $\left.10^{4}\right)$. Networks are first allowed to equilibrate into a 100-D virgin state (in the absence of antigen). Then they are stimulated for 25 days with a constant concentration of an antigen recognised 

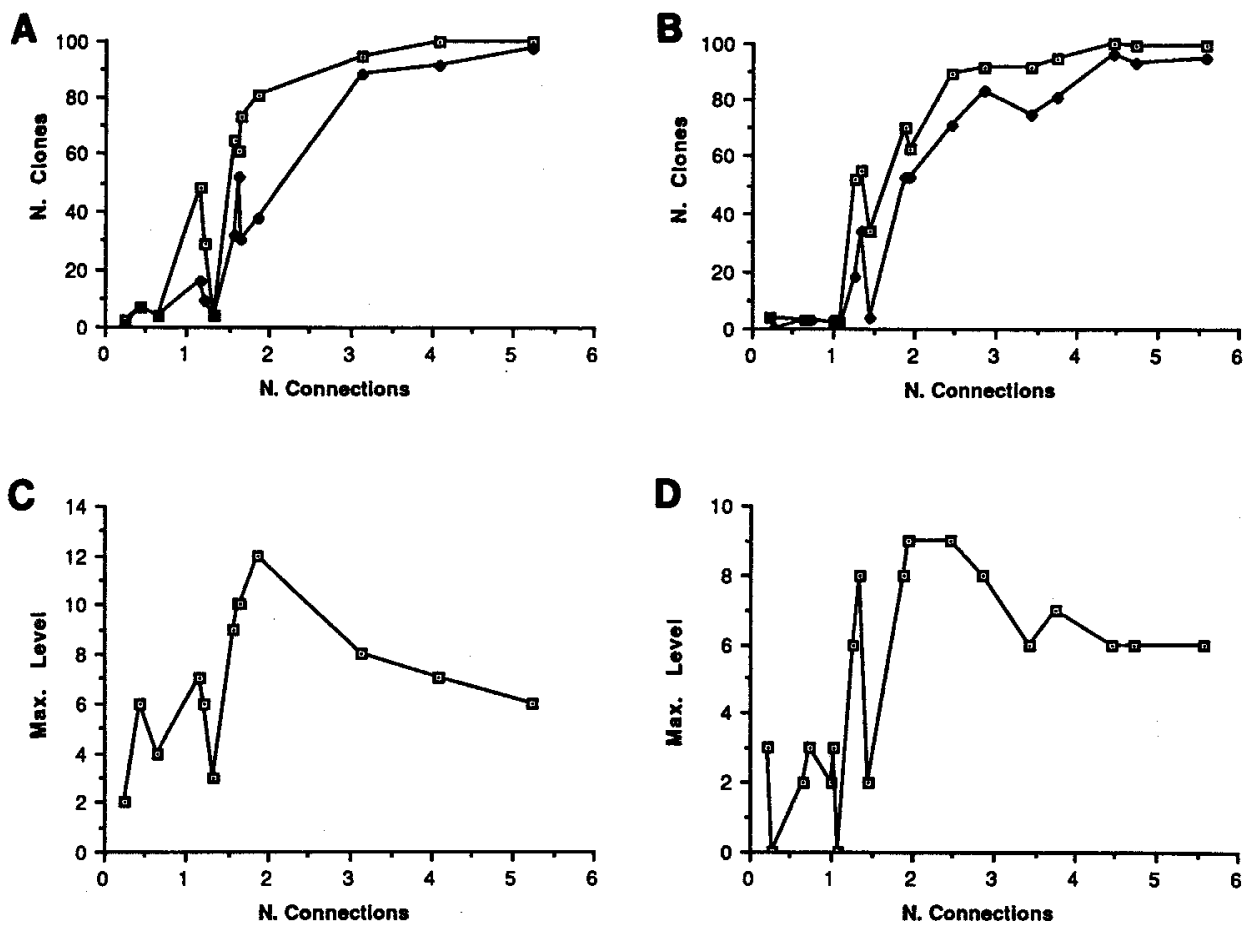

Figure 4. The effect of connectance in 100-D random and complementary matching (pattern) networks. (A) The actual dimension of the random network connected to the antigen ( $\square$ ) and the number of clones that actually switch (to immune or suppressed) following antigenic stimulation $(\diamond)$. (B) The same for the pattern networks. (C) and (D) The maximum antibody level reached by the idiotypic signal in random (C) and pattern (D) networks.

by only one clone (with at least one connection to the network). Two typical examples are shown in Fig. 5 (one around $(n c=1.04)$ and one slightly above ( $n c=1.36$ ) the Erdos and Renyi $E / N=1 / 2$ threshold). In the equilibrium that is finally reached we score: (1) the "effective dimension" of the network, i.e. the actual number of clones that are connected to the clone stimulated by antigen; (2) the number of clones that are actually affected, i.e. that have left the virgin state; (3) the depth of signal propagation, i.e. the maximum idotypic level affected. The first score (1) measures the above-mentioned Erdos and Renyi property; the other two link this with "absence of fading".

The effective dimension (Figs 4A and B) of our 100-D systems does indeed switch from low to high- $D$ interconnected networks around a connectance of one idiotypic partner per clone $(E / N=1 / 2)$. This switch is smoother in 50-D and steeper in 200-D networks (not shown); this explains why these results were less pronounced in our previous 50-D models (De Boer, 1988). The curve of the number of affected clones (Figs 4A and B: $\diamond$ ) is similar to (but somewhat smoother than) the "Erdos and Renyi" effective dimension curve (Figs 4A and 
A

$\log 10$ Pop.

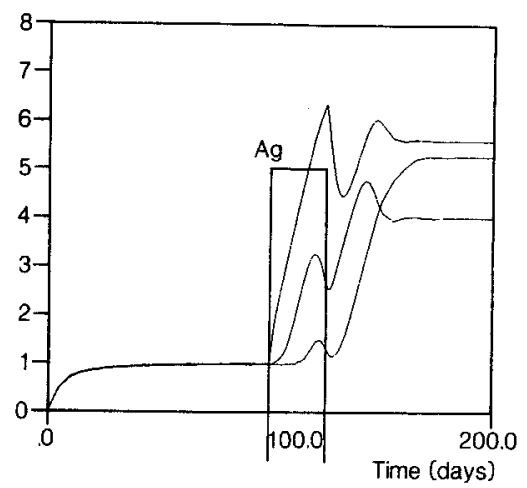

B

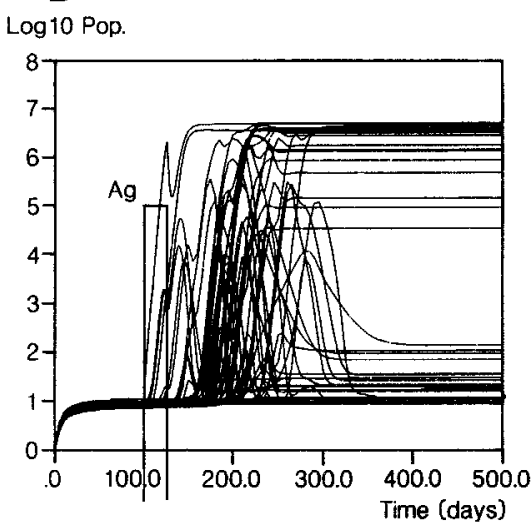

Figure 5. The behaviour of $100-D$ pattern networks (based on complementary matching). A network with on average 1.04 connections per clone (A), and (B) a network with 1.36 connections per clone. This is respectively just around and just above the Erdos and Reyni $(1959,1960)$ threshold of one edge per two nodes. The figure only depicts the clones that are actually connected to the network stimulated by antigen (all other clones remain virgin).

B: $\square)$. Whenever clones are connected to five or more idiotypic partners, the (first) antigen affects nearly all clones. Moreover $2 / 3$ of the affected clones are in the suppressed state at a connectance of five. As was demonstrated before (De Boer, 1988), the ratio of suppressed/immune clones increases if the connectance increases. Highly connected networks are thus highly unresponsive: almost all clones are suppressed. The maximum idiotypic level that is reached (Figs $4 \mathrm{C}$ and D) peaks around a connectance of two $(n c=2)$; in such networks signals penetrate deeply (here up to level 12). Maximum penetration depends on the dimension of the network. A 200-D network (with $n c=2$ ) reached a maximum idiotypic level of $17 ; 50-D$ networks reached lower levels. This is probably related to the fact that in low- $D$ networks pathways (connected structures) form closed loops sooner; as was mentioned above, this stops the signal. For a $10^{7}-D$ biotic immune system (for $n c \geqslant 2$ ) the maximum idiotypic level reached by any immune response is thus also expected to be very large.

We conclude that the "random" and "pattern" networks are very similar with respect to these connectivity properties. Thus, symmetric idiotypic networks that consist on one cell type (e.g. B cells) are statistically expected to form one interconnected structure whenever each clone, on average, has more than one idiotypic interaction. (Note that one interaction per clone is a minimal requirement for network interactions.) If this "interconnectance" is combined with our "percolation" results, we have to conclude that antigenic triggering of such a network will affect very many (i.e. most) of the clones of the network. 
The virgin state. Another connectivity threshold is the existence of the virgin state. By choosing the clonal influx of virgin cells small $\left(S_{i} \approx 10\right.$ cells day $^{-1}$ ), we deliberately kept the $N-D$ network virgin state devoid of idiotypic interactions. Virgin anti-idiotypic populations are thus too small to evoke idiotypic proliferation. In combination, a sufficiently large number of antiidiotypic clones should however be able to initiate proliferation in the virgin state (e.g. De Boer, 1988). This occurs more easily (i.e. at a lower connectance) if the influx of virgin cells is somewhat increased. To illustrate the effect of connectivity on the preservation of the virgin state we set $S_{i} \approx 50$ (we can only estimate the order of magnitude of such a parameter).

Around a connectance of 10 idiotypic partners per clone $(n c=10)$ networks suddenly lose their virgin state, which means that clones switch to immune or suppressed without previous exposure to antigen (Fig. 6). None of the clones remains in the virgin state. This is a form of autonomous network activity. Note that this exact value of $n c=10$ is parameter dependent, i.e. depends on the $S_{i} / P_{1}$ ratio. If $S_{i} \approx 10$ cells day ${ }^{-1}$, the system switches around $n c=50$; if $S_{i} \approx 100$ it switches around $n c=2$. The location of this threshold is, however, independent of the network dimension (De Boer, 1988): it is the sum of the idiotypic partners to which a clone responds. If antigen is introduced into such a highly connected system, the network appears unresponsive. Clones either fail to react because they are suppressed, or because they are already immune. Again the percentage of suppressed clones in this state increases as a function of connectance (not shown).

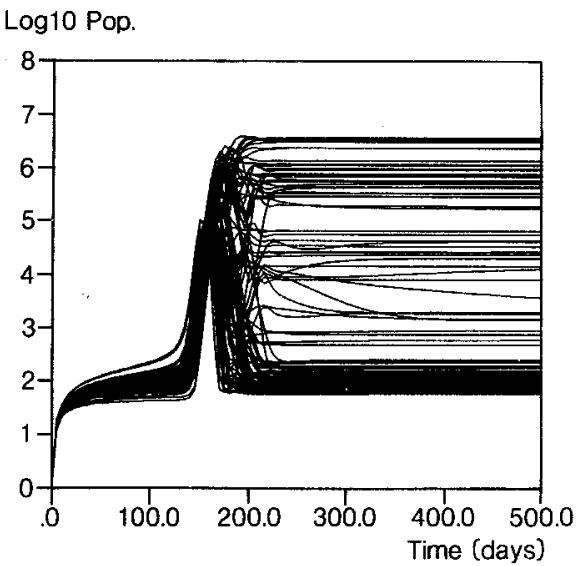

Figure 6. The neonatal state of a random 100-D network with a connectance of 10 connections per clone. We increased the influx of virgin cells $\left(5 \times\right.$, i.e. $S_{i} \approx 50$ cells per clone per day). 
We conclude that highly connected networks: (1) do not have a virgin state; (2) are unresponsive to external antigens; (3) display autonomous activity, i.e. clones switch to immune states in the absence of external antigens. We speculate that these results account for the differences reported to exist between highly connected neonatal networks (Holmberg et al., 1984, 1986; Vakil and Kearny, 1986) and weakly connected adult networks (Pollok et al., 1982; Holmberg et al., 1986). The former do indeed display autonomous activity (Pereira et al., 1986). We have demonstrated here that this marked difference an be accounted for solely by the (possibly gradual) difference in the connectance of the two types of networks.

Interpretation. We think that our results, i.e. the absence of suppression and the extensive percolation of idiotypic signals, suggest that immune systems do not function as profound idiotypic networks. The extensive percolation takes too much time and affects too many clones. By defining a minimal model we have maximally avoided the incorporation of ambiguous assumptions. Thus, because biotic immune systems are most probably based on a similar set of incorporated processes, our results might be general and not typical only of the models described here. It might, however, be argued that our results are artefacts arising from our simplifications. We therefore test the robustness of our results for the most obvious extensions of our model, i.e. "long-range inhibition", antibody molecules, and helper T cells. Incorporation of "longrange inhibition" and antibody molecules is relatively easy, and will be analysed in the next two sections; incorporation of helper $T$ cells is more difficult, and will be reported elsewhere (De Boer and Hogeweg, submitted).

4.3. Long-range inhibition and short-range activation. Segel and Perelson (1988) apply a general rule from theoretical biology in order to develop an interesting "shape-space" model of the idiotypic network. The incorporation of "long-range inhibition" and "short-range activation" in a model promotes instability, and hence pattern formation. It is therefore conceivable that our results hinge upon our assumption that the range of our stimulatory and inhibitory idiotypic interactions is identical. We here test the robustness of our "extensive percolation" results by allowing inhibitory interactions to be more general than stimulatory interactions. Intuitively, general suppression might indeed shut off the paths along which the idiotypic signal percolates into the network. Or as Segel and Perelson (1989) put it:

\footnotetext{
"Intuitively it is reasonable to assume that short range activation can give rise to a regular array of peaks from areas of local activation surrounded by more dispersed annuli of inhibition."
}

Additionally, an immunological argument can be made: stimulatory interactions require crosslinking of the $\mathrm{B}$ cell receptors, i.e. extensive binding by anti- 
idiotypic receptors, whereas it is conceivable that this crosslinking (i.e. activation) process may be inhibited (blurred) by low-affinity binding of many other anti-idiotypes.

We thus use two affinity matrices, one for activation (A) and one for the, more general, inhibition (B). The A matrix is identical to that used in the "pattern" networks; the B matrix can simply be made by using a smaller "mask" in the complementary matching algorithm. We here use a mask of 7 sites for the B matrix, and 8 sites for the A matrix. Note that the A matrix is always included in the $\mathbf{B}$ matrix, i.e. if two clones are able to activate each other (i.e. $\mathbf{A}_{i j}>0$ ), then they are necessarily able to inhibit each other's crosslinking process (i.e. $\mathbf{B}_{i j} \geqslant \mathbf{A}_{i j}$ ). Moreover, note that both matrices remain symmetric. For the above masks the connectivity of the $\mathbf{B}$ matrix is generally ten times higher than that of the $\mathbf{A}$ matrix. In order to incorporate "long-range inhibition" we implement the B matrix in our model:

$$
\begin{gathered}
\alpha l d_{i}=\sum_{j=1}^{N} \mathbf{A}_{i j} X_{j}, \\
\beta l d_{i}=\sum_{j=1}^{N} \mathbf{B}_{i j} X_{j}, \\
G\left(X_{i}, A g_{i}, \alpha l d_{i}, \beta l d_{i}\right)=\frac{A g_{i}+\alpha l d_{i}}{P_{1}+F X_{i}+A g_{i}+\alpha l d_{i}} \times \frac{P_{2}}{P_{2}+\beta l d_{i}}, \\
\frac{\mathrm{d} X_{i}}{\mathrm{~d} t}=S_{i}-D X_{i}+P X_{i} G\left(X_{i}, A g_{i}, \alpha l d_{i}, \beta l d_{i}\right) .
\end{gathered}
$$

Typical examples of such networks are shown in Fig. 7. Figure 7A shows the same, weakly connected, network as was displayed in Fig. 5B (i.e. the A matrices are identical: $n c=1.36$ ). This "long-range inhibition" network also displays extensive percolation: similar to what was described in Fig. 5B (see Fig. 4B), 35 of the 55 clones that were actually connected to the clone stimulated by antigen are affected by the signal (i.e. switch to an immune or suppressed state). Additionally, $33 \%$ of the 100 clones are finally more or less suppressed by the long-range inhibition ( $n c=9.72$ in the $\mathbf{B}$ matrix). Moreover, the network behaviour seems rather chaotic: the system oscillates irregularly and only settles into an equilibrium after two years. Another example of such "erratic" behaviour and slow settlement ( $>3$ years) into an equilibrium state is shown in Fig. 7B. This figure shows a network based on an average of 2.86 stimulatory and 21.2 inhibitory interactions per clone. Without the "longrange inhibition" this network (see Fig. 4B) managed to settle into an 
equilibrium in about 300 days. Networks below the connectivity threshold $(n c=1)$, by contrast, do behave very similarly to those of Section 4.2 . We conclude that, above the $n c=1$ threshold, "long-range inhibition" networks (1) can only slowly settle in equilibria (2) seem chaotic, and (3) also display extensive percolation.

A

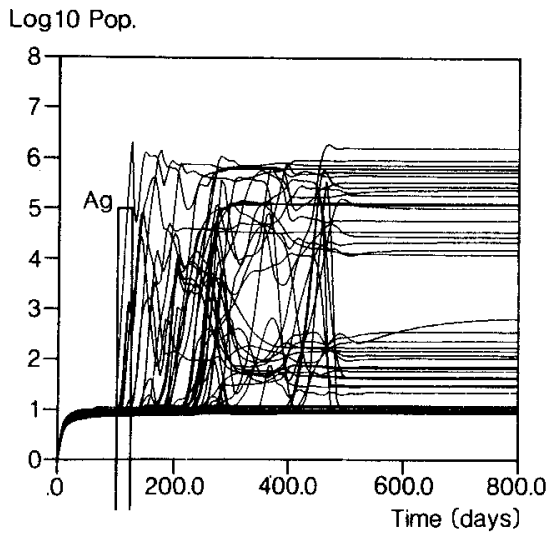

B

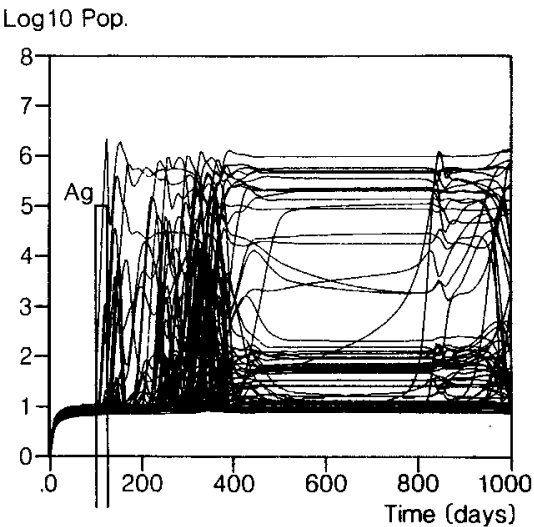

Figure 7. The behaviour of "longe-range inhibition" networks. (A) Each clone has on average 1.36 stimulatory interactions (i.e. the $\mathbf{A}$ matrix is identical to that of the network displayed in Fig. 5B) and on average 9.72 inhibitory interactions. The 100$D$ system also percolates extensively and takes about twice as long to settle in a stable state. (B) A similar network based on an average of 2.86 stimulatory and 21.2 inhibitory interactions per clone. The seemingly chaotic behaviour is very pronounced in these "longe-range inhibition" networks.

The explanation for these counter-intuitive results is the fact that our networks do not discriminate between "activators" (e.g. helpers) and "inhibitors" (e.g. suppressors). Whether a clone stimulates or inhibits its idiotypic partners depends on the local circumstances. Hence, the (long-range) inhibition of an immune (i.e. inhibitory) clone will transform that clone into a suppressed (i.e. stimulatory) clone. Thus "long-range inhibition" of immune clones implies "long-range activation". Our idiotypic network cannot fully discriminate between "inhibition" and "activation". Additionally, these networks are more chaotic because the "long-range" interactions disturb stable equilibria that were attained at previous idiotypic levels. Thus, through the inhibition, idiotypic interactions at previous levels switch to other ratios, which locally initiates the percolation of new idiotypic signals.

We conclude that the incorporation of "long-range inhibition" cannot prevent the "extensive percolation", but adds an additional "unreasonable implication", i.e. "extensive oscillations". We think that the type of chaos displayed in Fig. 7 cannot be characteristic for immune systems. Following 
antigenic stimulation the network oscillates unpredictably for periods of several years. It thus seems very likely that the clones that have to remain immune to the original antigen also become involved, and hence switch to suppressed states for extended periods of time (i.e. for months). Antigen specific immunity would thus become a rather chaotic phenomenon which switches on and off in time, until it unpredictably settles into "immune" or "suppressed". We are aware of the fact that some of the theoreticians and experimentalists think that immune networks do function by means of chaotic behaviour; we however think that this idea is altogether too "romantic". At least, we fail to see how this type of chaotic behaviour can be applied to our questions regarding antigen specific immunity and/or suppression.

4.4. Results: incorporation of long-lived antibodies. In the above models we simply lumped B-cells and their antibodies into one population $(X)$. Idiotypic communication between populations of $\mathbf{B}$ cells however occurs via their respective antibodies. Because the life-span of cells and antibodies is quite different (most B cells are short-lived and antibodies generally are long-lived) (Jerne, 1984), and because B cells proliferate before they produce antibodies (Melchers and Anderson, 1986), it seems important to complicate our model in order to incorporate antibody production. We now distinguish populations of cells $\left(X_{i}\right)$ from antibodies $\left(A b_{i}\right)$ :

$$
\begin{gathered}
\alpha l d_{i}=\sum_{j=1}^{N} \mathbf{A}_{i j} A b_{j}, \\
\frac{\mathrm{d} A g_{i}}{\mathrm{~d} t}=-\frac{K A g_{i} A b_{i}}{K_{1}+A b_{i}}, \\
\frac{\mathrm{d} A b_{i}}{\mathrm{~d} t}=M X_{i} \frac{A g_{i}+\alpha l d_{i}}{M_{1}+F X_{i}+A g_{i}+\alpha l d_{i}}-E A b_{i}-C A b_{i} \alpha l d_{i} .
\end{gathered}
$$

Equations (2) and (3) of our original model remain identical. Idiotypic stimulation and inhibition of cells now ocurs via the total of anti-idiotypic antibodies $\left[\alpha l d_{i}\right.$, equation $\left.\left(1^{\prime \prime}\right)\right]$. Antigen [equation ( $\left.\left.4^{\prime \prime}\right)\right]$ is now eliminated by antibodies. Antibodies [equation (5)] are produced by cells if these are sufficiently $\left(M_{1}\right)$ stimulated by antigen $\left(A g_{i}\right)$ and/or the anti-idiotypic antibodies $\left(\alpha l d_{i}\right)$. This interaction is again buffered $(F)$. Each fully activated cell produces $M$ antibody molecules; each molecule has a life-time of $1 / E$ days. In the present model it is possible to incorporate antibody removal by the formation of antibody complexes (at a rate $C$ ).

Parameters. The setting of the new parameters is relatively easy. We scale the antibody concentration into units produced per cell, i.e. $M=1$. Antibodies 
are relatively long-lived. The half-life of $\operatorname{Ig} M$ antibodies is about two days, that of $I g G$ 4-8 days (Vieira and Rajewsky, 1988). We here take a lifespan of roughly five days $(E=0.2)$; a life-span of about 10 days gives similar results (not shown). We will first ignore complex formation $(C=0)$. B cells differentiate into antibody-producing cells (plasma cells) after a number of proliferation cycles. This can be incorporated by setting $M_{1}>P_{1}$. Both models [i.e. equations (1)-(3) and equations (1') and (2)-(4)] are however most equivalent if $M_{1} \ll P_{1}$, i.e. if the antibody concentration is proportional to the (cell) population density. Because the antibodies live five times longer than the $\mathrm{B}$ cells, such "equivalent" antibody concentrations are generally 5 times higher than the B cell population density. By rescaling $P_{1}$ to $5 \times 10^{3}, P_{2}$ to $5 \times 10^{6}$, and $K_{1}$ to $5 \times 10^{5}$ (i.e. by a 5 -fold increase) we can easily compensate for this difference; this is "external equivalence" (Irvine and Savageau, 1985).

Figure 8A displays the 0-isoclines of a two-dimensional network of this "antibody" model for these (external) equivalence conditions (i.e. for $M_{1}=1$, $\left.P_{1}=5 \times 10^{3}, P_{2}=5 \times 10^{6}\right)$. In order to perform this two-dimensional graphical analysis (which is directly comparable to Fig. $2 A$ ) of this 4-D model, we made a quasi-steady-state assumption for the antibodies [equation (4)]. (We make this assumption just for this comparative static analysis; dynamically it is not justified, see below.) The isoclines and hence the equilibrium points are very similar to those of Fig. 2. The only difference is the population level to which clones are suppressed: clones remain larger here (even lower $M_{1}$ values give identical results). The suppression is reduced because a clone that suppresses another clone requires continuous idiotypic stimulation (by antibodies produced by the suppressed clone) in order to be able to produce its (suppressive) antibodies. Moreover, suppressive clones are typically large and hence require quite vigorous stimulatory interactions (due to the buffering). However, since the equilibrium states are very similar to those of the previous model, similar switching behaviour is to be expected.

It seems more interesting however to incorporate the empirical finding that B cells produce antibodies after a proliferation period, i.e. to set $M_{1}>P_{1}$. This again hardly affects the equilibrium states (see Fig. 8B, $M_{1}=5 \times 10^{4}$, $\left.P_{1}=5 \times 10^{3}, P_{2}=5 \times 10^{6}\right)$; only the region of attraction of the virgin state increases (which may be advantageous because it gets lost rather easily, Fig. 7). The effect of the $M_{1}$ value on the isoclines is analysed more gradually in Fig. 9. If antibody production starts too late (e.g. $M_{1} \gg 10^{6}$ ) the idiotypic proliferation region decreases; hence idiotypic interactions lose their effect. The balance of proliferation and differentiation (Grossmann, 1982) thus requires to be tuned accurately. If we do this $\left(M_{1}=5 \times 10^{4}\right)$, and if we stimulate this "quasi-steadystate system" with antigen it does indeed switch to the corresponding immune state. In this equilibrium the antibody concentration of the suppressed antiidiotypic $\left(\mathrm{X}_{2}\right)$ clone also suffices for activating the next (virgin) anti-anti- 

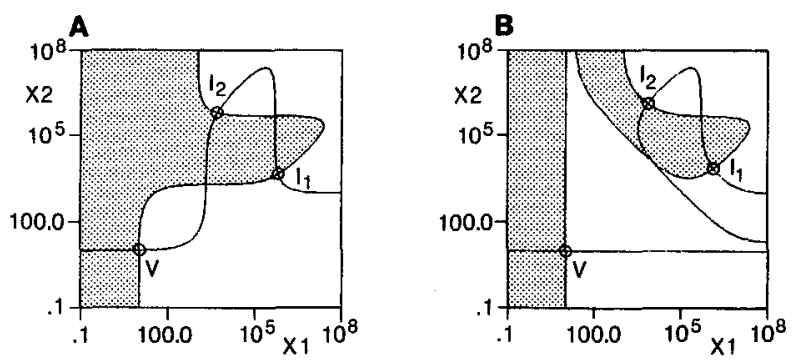

Figure 8. A two-dimensional network incorporating antibodies (for $A_{12}=1$ ). The isoclines are drawn for a quasi-steady-state assumption for the antibody equations [equation (4)]. (A) A system which is externally equivalent to that of Fig. 2 (i.e. $M_{1}=1, P_{1}=5 \times 10^{3}$, and $\left.P_{2}=5 \times 10^{6}\right)$. (B) A system in which proliferation precedes antibody production (i.e. $M_{1}=5 \times 10^{4}, P_{1}=5 \times 10^{3}$, and $P_{2}=5 \times 10^{6}$ ). The equilibria remain qualitatively the same to those of the previous model (Fig. 2).

idiotype $\left(X_{3}\right)$ clone. Signals thus still fail to fade. We conclude that, for a sufficiently low onset of antibody production (e.g. $M_{1} \approx 5 \times 10^{4}$ ), we have succeeded in incorporating "antibody production after proliferation" but have preserved the previous isoclines and steady states.

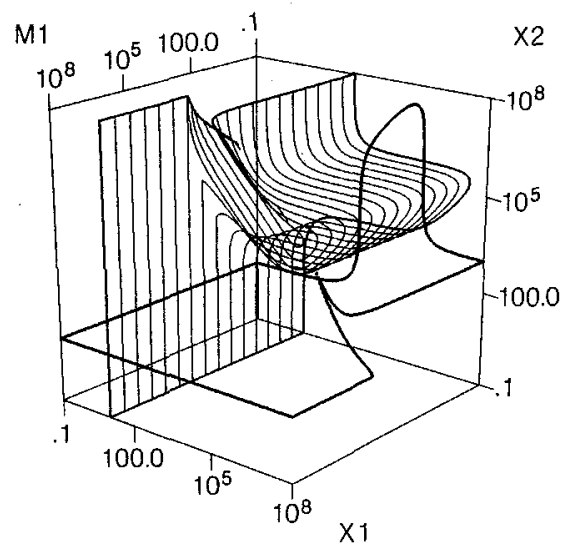

Figure 9. The $X_{1}^{\prime}=0$ and $X_{2}^{\prime}=0$ isoclines as a function of the onset of antibody production $\left(M_{1}\right)$. The immune states exist only if $M_{1}$ is sufficiently small.

However if we analyse this model dynamically, i.e. if we relax the quasi steady state assumption for the antibodies [equation (4)], totally different results are obtained. In the virgin state $A g_{1}$ is introduced in a dose of $10^{5}$ cells; this dose suffices for antibody production (i.e. $A g_{1}>M_{1}=5 \times 10^{4}$ ). Figure $10 \mathrm{~A}$ shows a time plot of the system behaviour: antigen is rejected but the systems switches from virgin to cyclic behaviour: it never settles in any (immunity) equilibrium. Figure 10B shows the same behaviour, now plotted in the $X_{1}$ vs 
$X_{2}$ (i.e. the idiotype vs anti-idiotype) state space. We conclude that although this model has similar equilibria, they are no longer attractive when antibodies are really made long-lived (i.e. by relaxation of the quasi-steady-state assumption). Thus the difference in the lifespan of cells and antibodies totally disrupts the neat switching behaviour of the previous model. Because in biotic immune systems antibodies and cells do have a different lifespan, the generation of memory phenomena by idiotypic interactions becomes even more questionable.
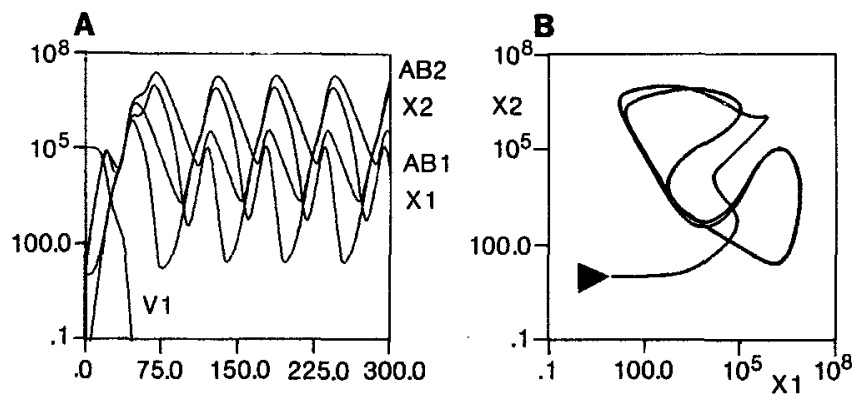

Figure 10. The behaviour of the antibody model (the quasi steady state assumption being relaxed). The two-dimensional network is stimulated with an antigen dose $A g_{1}=10^{5}$. Antigen is rejected but the network fails to switch and enters an infinite cycle which is displayed in time (A), and in the $X_{1}$ vs $X_{2}$ space (B).

The cycles take place in the complete absence of antigen: antigen was rejected around day 60 . Figure $10 \mathrm{~A}$ shows the explanation for the cycles: around day 60 the high and suppressive $A b_{1}$ concentration slowly decays (due to antibody turnover). This means that after a while the suppressive $A b_{1}$ concentration becomes stimulatory again (i.e. when $A b_{1}<P_{2}$ ). This reactivates idiotypic proliferation until the $A b_{1}$ concentration becomes inhibitory again. Again $A b_{1}$ slowly decays (due to turnover) and the cycle repeats itself. Thus, as was the case in the "long-range inhibition" model, clones switch from "stimulatory" to "inhibitory" by moving along the bell-shaped dose response curve. This type of switching is based on our most fundamental assumptions: (1) no distinction between "helpers" and "suppressors"; (2) the bell-shaped growth functions. Here, the switching (and cycling) is essentially determined by the autonomous slow decay of the antibodies.

Complex formation. The (possible) removal of antibodies by the formation of idiotypic anti-idiotypic antibody complexes is a process that reduces the intensity of idiotypic interactions between cells. This occurs when antibody concentrations are large (compare our bell-shaped dose response function). The rate of complex formation is determined by our $C$ parameter ( $C$ was zero above). If we replace our bell-shaped curve by a saturation function (i.e. set $P_{2}$ 
to $\infty$ ), and incorporate complex formation (i.e. set $C>0$ ), we obtain Fig. 11 . This "antibody complexes" model generally has only one virgin state and no immune states (if $C$ is extremely small (e.g. $10^{-8}$ ) the system has one immune state at unrealistically high populations $\left(10^{10}\right.$ cells $\left.)\right)$. We conclude that complex formation on its own cannot account for interesting switching behaviour. Furthermore, by complex formation alone, a large idiotypic population (antibody and/or cells) can never "suppress" a small anti-idiotypic population just because the latter is too small to remove the former.

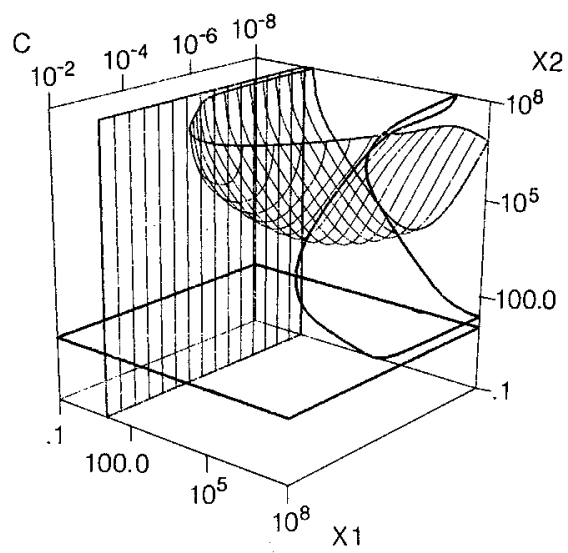

Figure 11. The $X_{1}^{\prime}=0$ and $X_{2}^{\prime}=0$ isoclines as a function of the rate of antibody complex formation $(C)$ for $P_{2}=\infty$ (i.e. when complex formation is the only inhibitory interaction in the system). For realistic population densities this system never has immune states.

Complex formation can also be combined with our bell-shaped dose response curve (i.e. keep $P_{2}=5 \times 10^{6}$ and set $C>0$ ). Such models only have multiple stable points if $C$ is sufficiently small, i.e. whenever complex formation plays a sufficiently small role. At very high antibody concentrations however, such small $C$ values give rise to complex formation. Thus suppressive antibody levels are more quickly reduced to stimulatory levels. Hence complex formation accelerates the cycle displayed in Fig. 10, and fails to play a stabilizing role in the present models.

5. Discussion. We have analysed a simple model which incorporates only fundamental idiotypic network assumptions. This model nevertheless displayed unexpected and/or unreasonable behaviour. We concluded that, if idiotypic networks switch from virgin to immune states, such switches propagate infinitely into the network. Moreover, the idiotypic control of proliferation seems impossible because proliferating clones suppress their antiidiotypic suppressors. Highly connected networks display autonomous 
behaviour but are unresponsive to foreign antigens. Long-range inhibition cannot solve these "percolation problems". The simple difference in the lifespan of antibodies and cells generates complex cycling behaviour. This behaviour is governed by the autonomous slow decay of antibodies and totally disrupts the fine tuning of the idiotypic interactions.

The absence of interesting network behaviour in our model contrasts strongly with the abundance of experimental results on idiotypic networks. This discrepancy might suggest that our assumptions are wrong or, more likely, insufficient. Alternatively, however, we might question the validity of the experimental results. In our model, with all its "unreasonable implications" we can easily obtain "interesting results" by "experimental manipulation" of the system. In the model idiotypic control of proliferation can be achieved by the introduction of a large dose of anti-idiotypic antibody (or cells) (De Boer and Hogeweg, 1988). Such manipulation eliminates the "natural" advantage of the clone responding to antigen. Moreover, we can manipulate the neonatal repertoire (i.e. the equilibrium state) of highly connected networks by introducing one of the clones (or antibodies) during the neonatal development. Our "manipulated" network however remains unresponsive to most (but different) antigens, and hence remains non-functional. These examples show that "interesting" empirical data do not necessarily prove the functionality of the network, but can be a consequence of the experimental manipulation.

Other experimental data, by contrast, confirm our idea that immune systems do not function as a profound network of idiotypic interactions (reminiscent of neural networks). These data suggest that the network is not "open ended" but that idiotypic interactions remain confined to specific and limited structures. In these experimental systems (Wikler et al., 1979) it was shown that $A b 3$ is idiotypically related to $A b 1$ (although $A b 3$ does not bind antigen), and that $A b 4$ resembles $A b 2$. Thus, experimental data were recently interpreted in terms of "flat" networks, e.g. the "broken mirror" hypothesis (Urbain, 1986) or Jerne's (1984) system of "preferred partners", and not in terms of profound networks. This accords with our results. However, such structures do not arise spontaneously (see e.g. our "pattern" networks), i.e. idiotypic profiles are expected to diverge. We therefore plan to investigate the type of idiotypic interactions that would generate such (or similar) confined immune network reactions.

In conclusion, the present results contradict our intuitive expectations about the functioning of this simple (fundamental) network. This can be taken as another argument supporting the idea that (biotic) idiotypic networks are nonfunctional (Cohn, 1986; Langman and Cohn, 1986). Alternatively, the results can be taken as an argument that the idiotypic network must be more complex than we assume in our models. This would certainly be important because it would pinpoint the additional network assumptions that have to be made if 
idiotypic network theory is to adequately account for the functioning of immune systems. We have here tested two such complications, i.e. (1) "longrange inhibition" and (2) the difference between the lifespan of cells and antibodies, and have concluded that the additional complexity can only hamper the functioning of the network.

The incorporation of T-B cell cooperation is the next obvious extension of the our simple B cell idiotypic network model. Production of anti-idiotypic antibodies has indeed been demonstrated to depend on helper $T$ cells (Trenker and Riblet, 1975). Elsewhere (De Boer and Hogeweg, submitted) we will analyse the role that helper $\mathrm{T}$ cells play in $\mathrm{T}-\mathrm{B}$ cell idiotypic networks. These models are however compliated. Firstly, for the T-B cell idiotypic interaction, one has to distinguish between (1) MHC-restricted helper T cells, and (2) helper $\mathrm{T}$ cell receptors that bind directly to B cell idiotypes. Secondly, we distinguish antigen-specific and idiotype-specific helper $T$ cells, Thirdly, we have to consider percolation pathways consisting of either B cell clones (each helped by a helper clone) or of mixtures of $B$ and $T$ cell clones. The most important conclusions are: (1) MHC restricted T-B idiotypic interactions are expected to be asymmetric; (2) symmetric and asymmetric helper inteactions may stop the percolation along the $A g-B_{1}-B_{2}-B_{3}$ pathway at level of the second B cell clone; (3) MHC-restricted helper interactions avoid percolation along $A g-B_{1}-T_{2}-B_{3}$ pathways. These results (De Boer and Hogeweg, submitted) suggest that for $B$ cell idiotypic interactions helper $T$ cell activation is of crucial importance. Furthermore, the results suggest that helper interactions fail to account for limited percolation, i.e. for localized network responses.

The sensitivity of idiotypic B-B interactions to helper $\mathrm{T}$ cell activation implies that (theoretical and experimental) network models that neglect helper $T$ cells can only be valid for helper-independent idiotypic interactions. Although it has never been demonstrated experimentally, B cell idiotypic interactions of the $I g M$ networks of early (i.e. developing) immune systems (Holmberg et al., 1984, 1986) are possibly helper independent. These early IgM networks are supposed to develop before T cells emerge (Martinez-A et al., 1988). Thus, if helper interactions are indeed so crucially important, the present B-B idiotypic network model would only be valid for the early $I g M$ network situation. Moreover, as was discussed at the end of Section 4.2, extensive percolation is probably realistic in these highly connected $I g M$ networks. The autonomous network activity (Fig. 6) does not have to account for antigenspecific immunity, but may, instead, play a role in the selection of B and/or T cell repertoires (Vakil and Kearny, 1986; Martinez-A et al., 1988; own unpublished results).

Because we robustly fail to find a solution for the unrealistic percolation problems that arise in the various idiotypic networks that we are analysing, we 
have become very critical of the hypothesis that the phenomenon of immunological memory is generated by (cognitive) state switches in profound networks of idiotypic interactions.

We thank Ms S. M. McNab for linguistic advice.

\section{LITERATURE}

Abbas, A. K. 1988. "A Reassessment of the Mechanisms of Antigen-Specific T-cell-Dependent Bcell Activation." Immunol. Today 9, 89 94.

Berek, C., G. M. Griffiths and C. Milstein. 1985. "Molecular Events During Maturation of the Immune Response to Oxazolone." Nature 316, 314-318.

Bernable, R. R., A. Coutinho, C. Martinez-A and P. A. Cazenave. 1981. "Immune Networks. Frequencies of Antibody-and Idiotype-Producing B cell Clones in Various Steady States." $J$. exp. Med. 154, 552-556.

Bona, C. A. and B. Pernis. 1984. "Idiotypic Networks." In Fundamental Immunology, W. E. Paul (Ed.), pp. 577-592. New York: Raven Press.

Cohn, M. 1986. "The Concept of Functional Idiotypic Network for Immune Regulation Mocks all and Comforts None." Ann. Immunol. (Inst. Pasteur) 137C, 64-76.

De Boer, R. J. 1983. GRIND: Great Integrator Differential Equations. Bioinformatics Group, University of Utrecht, The Netherlands.

- . 1988. "Symmetric Idiotypic Networks: Connectance and Switching, Stability, and Suppression." In Theoretical Immunology, A. S. Perelson (Ed.), Part Two, pp. 265-289. SFI Studies in the Science of Complexity, Vol. III. Reading, MA: Addison-Wesley.

-1989. "Information Processing in Immune Systems: Clonal Selection versus Idiotypic Network Models." In Theoretical Models for Cell to Cell Signalling, A. Goldbeter (Ed.). London: Academic Press.

and P. Hogeweg. 1986. "Interactions between Macrophages and T-lymphocytes: Tumor Sneaking Through Intrinsic to Helper T cell Dynamics." J. theor. Biol. 120, 331-351.

_ and 1987a. "Immunological Discrimination Between Self and Non-Self by Precursor Depletion and Memory Accumulation." J. theor. Biol. 124, 343-369.

and __ . 1987b. "Self-Nonself Discrimination due to Immunological Nonlinearities: the Analysis of a Series of Models by Numerical Methods." IMA J.Math. Appl. Med. Biol.4, $1-32$.

___ and - 1988. "Memory but no Suppression in Low-Dimensional Symmetric Idiotypic Networks." Bull. math. Biol. 51, 0.

_ ation: Conditions for Percolation."

Early, P., H. Huang, M. Davis, K. Calame and L. Hood. 1980. "An Immunoglobulin Heavy Chain Variable Region Gene is Generated from Three Segments of DNA: $V_{\mathrm{H}}, D$ and $J_{\mathrm{H}}$." Cell 19, 981-992.

Eichmann, K. 1974. "Idiotypic Suppression-I. Influence of the Dose and of the Effector Functions of Anti-Idiotypic Antibody on the Production of an Idiotype." Eur. J. Immunol.4, 296-302.

- and K. Rajewsky. 1975. "Induction of T and B cell Immunity by Anti-Idiotypic Antibody." Eur. J. Immunol. 5, 661-666.

Erdos, P. and A. Renyi. 1959. "On the Random Graphs 1, Vol. 6." Institute of Mathematics University of DeBreceniens, Debrecar, Hungary.

and - 1960. "On the Random Graphs, Publ. No. 5." Mathematics Institute of the Hungarian Academy of Science.

Farmer, J. D., N. H. Packard and A. S. Perelson. 1986. "The Immune System, Adaptation, and Machine Learning." Physica 22D, 187-204. 
Goldstein, B. 1988. "Desensitization, histamine release and the aggregation of $I g E$ on human basophils." In Theoretical Immunology, A. S. Perelson (Ed.), Part One, pp. 3-40. SFI Studies in the Science of Complexity, Vol. II. Reading, MA: Addison-Wesley.

Gottwald, B. A. and G. Wanner. 1981. "A Reliable Rosenbrock Integrator for Stiff Differential Equations." Computing 26, 355-360.

Gray, D. 1988. "Is the Survival of Memory B cells Dependent on the Persistence of Antigen?" In Adv. Exp. Med. Biol., in press.

Grossman, Z. 1982. "Recognition of Self and Regulation of Specificity at the Level of Cell Populations." Immunol. Rev. 79, 119-138.

Gunther, N. and G. W. Hoffmann. 1982. "Qualitative Dynamics of a Network Model of Regulation of the Immune System: a Rationale for the $\operatorname{Ig} M$ to $\operatorname{Ig} G$ switch." J. theor. Biol. 94, $815-855$.

Hardt, D. A., A. L. Wang, L. L. Pawlak and A. Nisonoff. 1972. "Suppression of Idiotypic Specificities in Adult Mice by Administration of Anti-Idiotypic Antibody." J. exp. Med. 135, 1293-1299.

Hebb, D. O. 1949. The Organization of Behavior. New York: Wiley.

Hoffmann, G. W. 1975. "A Theory of Regulation and Self Non-Self Discrimination in an Immune Network.” Eur. J. Immunol. 5, 638-647.

- 1979. "A Mathematical Model of the Stable States of a Network Theory of Self-Regulation." In Systems Theory in Immunology, C. Bruni, G. Doria, G. Koch and R. Strom (Eds), Vol. 32, pp. 239-257. Lecture Notes in Biomathematics. Berlin: Springer.

- 1980. "On Network Theory and H-2 Restriction." In Contemporary Topics Immunobio$\log y$, N. L. Warner (Ed.), Vol. 11, pp. 185-226. New York: Plenum Press.

_. 1986. "A Neural Network Model Based on the Analogy with the Immune System." $J$. theor. Biol. 122, 33-67.

Holland, J. H. 1986. "Escaping Brittleness: the Possibilities of General Purpose Learning Algorithms Applied to Parallel Rule-Based Systems." In Machine Learning: An Artificial Intelligence Approach, R. S. Michalski, J. G. Carbonell and T. M. Mitchell (Eds), Vol. II, pp. 593-623. Los Altos: Morgan Kauffman.

Holmberg, D., S. Forsgen, F. Ivars and A. Coutinho. 1984. "Reactions Among IgM Antibodies Derived from Normal Neonatal Mice." Eur. J. Immuno: 14, 435 441.

- G. Wennerstrom, L. Andrade and A. Coutinho. 1986. "The High Idiotypic Connectivity of "Natural" Newborn Antibodies is not found in the Adult Mitogen-Reactive B Cell Repertoires." Eur. J. Immunol. 16, 82-87.

-1987. "High Connectivity, Natural Antibodies Preferentially use 7183 and QUPC $52 V_{\mathbf{H}}$ Families." Eur. J. Immunol. 17, 399-403.

Hopfield, J. J. and D. W. Tank. 1986. “Computing with Neural Circuits: a Model." Science 233, 625-633.

Irvine, D. H. and M. A. Savageau. 1985a. "Network Regulation of the Immune Response: Alternative Control Points for Suppressor Modulation of Effector Lymphocytes." $J$. Immunol. 134, 2100-2116.

Jerne, N. K. 1974. "Towards a Network Theory of the Immune System." Ann. Immunol. (Inst. Pasteur) 125C, 373-389.

. 1984. "Idiotypic Networks and Other Preconceived Ideas." Immunol. Rev. 79, 5-24.

Kauffman, S. A. 1986. "Autocatalytic Sets of Proteins." J. theor. Biol. 119, 1-24.

Langman, R. E. and M. Cohn. 1986. "The 'Complete' Idiotypic Network is an Absurd Immune System." Immunol. Today 7, 100-101.

Lawler, A. M., P. Sin and P. J. Gearhart. 1987. "Adult B-cell Repertoire is Biased Toward Two Heavy-Chain Variable-Region Genes that Rearrange Frequently in Fetal pre-B cells." Proc. Natn. Acad. Sci. U.S.A. 84, 2454-2458.

Martinez-A, C., P. Pereira, M. L. Toribo, M. A. R. Marcos, A. Bandeira, A. De la Hera, C. Marquez, P-A. Cazenave and A. Coutinho. 1988. "The Participation of B cells and Antibodies in the Selection and Maintenance of T cell Repertoires." Immunol. Rev. 101, 191-215. 
Melchers, F. and J. Anderson. 1986. "Factors Controlling the B-cell Cycle." Ann. Rev. Immunol. 4, 13-36.

NAG. 1984. Numerical Algorithms Group, Oxford, U.K.

Novotny, J., M. Handschumacher and R. E. Bruccoleri. 1987. "Protein Antigenicity: a Static Surface Property." Immunol. Today 8, 26-31.

Pereira, P., L. Forni, E. L. Larsson, M. Cooper, C. Heusser and A. Coutinho. 1986. "Autonomous Activation of B and T cells in Antigen-Free Mice." Eur. J. Immunol. 16, 685-688.

Perelson, A. S. 1988. "Towards a Realistic Model of the Immune Network." In Theoretical Immunology, A. S. Perelson (Ed.), Part Two, pp. 377-401. SFI Studies in the Science of Complexity, Vol. III. Reading, MA: Addison-Wesley.

1984. "Some Mathematical Models of Receptor Clustering by Multivalent Ligands." In Cell Surface Dynamics: Concepts and Models, A. S. Perelson, C. DeLisi, and F. W. Wiegel (Eds), pp. 223-275. New York: Marcel Dekker.

Pollok, B. A., A. S. Bhown and J. F. Kearny. 1982. "Structural and Biological Properties of a Monoclonal Auto-Anti-(Anti-idiotype) Antibody." Nature 299, 447-449.

Trenker, E. and R. Riblet. 1975. "Induction of Antiphosorylcholine Antibody Formation by Anti-Idiotypic Antibodies." J. exp. Med. 142, 1121-1132.

Segel, L. A. and A. S. Perelson. 1988. "Computation in Shape Space: A New Approach to Immune Network Theory." In Theoretical Immunology, A. S. Perelson (Ed.), Part Two, pp. 321-343. SFI Studies in the Science of Complexity, Vol. III. Reading, MA: AddisonWesley.

__ _ and _ _ 1989. "Explanation of a Paradoxical Instability Caused by Relatively Short Range Inhibition." SIAM J. appl. Math, in press.

Urbain, J. 1986. "Idiotypic Networks: a Noisy Background or a Breakthrough in Immunological Thinking? The Broken Mirror Hypothesis." Ann. Immunol. (Inst. Pasteur) 137C, 57-64.

Vakil, M. and J. F. Kearny. 1986. "Functional Characterization of Monoclonal Auto-AntiIdiotype Antibodies Isolated from the Early B cell Repertorie of BALB/c Mice." Eur. $J$. Immunol. 16, 1151-1158.

Varela, F. J., A. Coutinho, B. Dupire and N. N. Vaz. 1988. "Cognitive Networks: Immune, Neural, and Otherwise." In Theoretical Immunology, A. S. Perelson (Ed.), Part Two, pp. 359-375. SFI Studies in the Science of Complexity, Vol. III. Reading, MA: AddisonWesley.

Vieira, P. and K. Rajewsky. 1988. "The Half-Lives of Serum Immunologlobulins in Adult Mice." Eur. J. Immunol. 18, 313-316.

Weisbuch, G. 1989. Proceedings of "Theories of Immune Networks" Workshop. Jerusalem, May 1988. In Lecture Notes in Biomathematics, in press.

Wikler, M., J-D. Franssen, C. Collignon, O. Leo, B. Mariamé, P. Van de Walle, D. De Groote and J. Urbain. 1979. "Idioptypic Regulation of the Immune System." J. exp. Med. 150, 184-195. 